\title{
Can We Replace Liver Biopsy with Non-Invasive Procedures?
}

\author{
Carmen Fierbinteanu Braticevici¹, Raluca Papacocea², \\ Laura Tribus ${ }^{1}$ and Anca Badarau ${ }^{2}$ \\ ${ }^{1}$ Medical Clinic II and Gastroenterology, University Hospital Bucharest, \\ ${ }^{2}$ Physiology Department I, \\ "Carol Davila" University of Medicine and Pharmacy Bucharest, \\ Romania
}

\section{Introduction}

Liver fibrogenesis is the consequence of all hepatic lesions, regardless of etiology. Progressive scars, which are the response to constant liver injuries lead to cirrhosis, disorganize the normal liver architecture through fibrosis bands, parenchyma nodules and blood vessel distortions. For this reason, liver fibrosis is a central parameter which expresses the severity of liver diseases, regardless of etiology; it is a key element which predicts the evolution of liver diseases towards cirrhosis.

Irrespective of the cirrhosis etiology, the clinical evolution is similar, towards major complications such as: portal hypertension and hepatocellular insufficiency. Due to these complications, cirrhosis is the seventh death cause within general mortality. Cirrhosis also encourages the development of hepatocellular carcinoma, an aggressive neoplasm which causes death in a few months in the absence of early diagnosis. That is why liver fibrosis is a major criterion in initiating the etiologic treatment of chronic liver diseases.

A long time ago, liver fibrosis was deemed irreversible. This axioma has been discarded for quite some time; at present, fibrosis is considered to be a bidirectional dynamic phenomenon: fibrogenesis and fibrinolysis. Thus, it is possible to reshape the scar healing tissue. Due to this dynamic process, it is necessary to quantify fibrosis in order to set the therapeutic decision and especially to monitor the efficiency of anti fibrotic treatments.

There are several methods to diagnose liver fibrosis: liver biopsy, serum biomarkers, breath tests and hepatic elastography. Liver biopsy is currently essential in diagnosis of inflammatory and metabolic liver diseases. It provides particularly invaluable informations for disease diagnosis and patient monitoring.

The role of liver biopsy is still a controversial issue being an invasive method with wellknown risks and complications. Although liver biopsy is considered the golden standard for chronic liver diseases, it is worth mentioning that the histological tested fragment is however a small part of the liver, and the scar lesions, which are secondary to chronic inflammatory processes are unevenly distributed in the liver mass. Thus, liver biopsy is a method neither ideal nor sufficient to diagnose and determine the stage of liver fibrosis. In this context, a noninvasive method to assess liver fibrosis is more than welcome. 
Noninvasive tests can be classified in several ways based on the modality of the test (serum blood tests or imaging) or the constituents of the tests (direct markers versus indirect markers of fibrosis). With the evolution of noninvasive tests, the performance can improve particularly with the use of combination or serial noninvasive tests.

In this chapter the role of liver biopsy and non-invasive tests to assess liver fibrosis and to manage the treatment of patients with chronic liver disease is discussed.

\section{Liver biopsy}

Liver biopsy was first described by Paul Ehrlich, 130 years ago, as a diagnostic tool of the chronic liver disease. It can be performed using blind percutaneous technique, computer tomography or ultrasound-guided, transjugular, open surgical, laparoscopic and even endoscopic approaches.

Microscopic examination of liver tissue often provides the definitive diagnosis and leads to effective management of the liver disorders. Because fibrosis is tissue damage, biopsy is by definition the only direct tool to assesses liver fibrosis.

\subsection{When is liver biopsy useful?}

The major indication for performing a liver biopsy is to clarify the nature of liver disease. In some instances, liver biopsy is performed to establish the effect of the treatment in known liver disease. In chronic viral hepatitis, the knowledge of liver fibrosis stage is important for prognosis and for decision about the antiviral treatment. Significant fibrosis $(F \geq 2)$ in these patients is an indication for antiviral treatment.

In patients with alcoholic liver diseases, the clinical features and the values of serum aminotransferases are poorly correlated with the liver diseases stage and the long-term prognosis strictly depends on the severity of histological lesions. In alcoholic liver disease, the biopsy can reveal fatty liver infiltration, large shape of hepatocytes, the presence of Mallory bodies, or polimorfonuclear infiltrates in portal spaces, and also histological features specific to cirrhosis. On the other side, fatty liver infiltration occurs in various etiologic situations: obesity, diabetes, consumption of alcoholic beverages, chronic HCV infection and the liver biopsy may provide distinct etiologic information.

In patients with chronic hepatitis $C$ there is a low correlation between symptoms, serum aminotransferases and histological modifications; thus, serum aminotransferases may have normal values, but liver histology is suggestive for severe fibrosis or cirrhosis. Liver biopsy ensures a certain diagnosis in more than $90 \%$ of the patients with unexplainable increase of serum aminotransferases. In the same time, it is useful in evaluating infectious complications, drug toxicity or the reoccurrence of the primary disease in the transplanted liver.

In patients who present space replacing liver formations, the indication of liver biopsy is still debatable. It is useful to establish the origin of metastasis when the primary tumor is not known, to confirm the metastases in case of diagnosed cancers before and after surgical resection. It is also able to certify the benign nature of liver formations.

In patients with nonresected hepatocellular carcinoma, echographic guidance allows specific therapeutically approach like: alcoholization, radiofrequency, chemoembolization. In conclusion, liver biopsy is recommended in diagnosis of:

Infiltrative liver diseases:tuberculosis, sarcoidosis, lymphoma, amyloidosis;

Hereditary disorders: Wilson disease (determining the amount of intrahepatocyte copper deposits), hemochromatosis (determining the amount and quality of intrahepatocyte iron deposits), alpha-1 antitrypsin deficiency; 
Liver cholestasis: primary billiary cirrhosis, primary sclerosing cholangitis;.

Diagnostic uncertainty (abnormal liver tests with no clear etiology: the increase of serum aminotransferases, bilirubin, alkaline phosphatase or Yglutamiltranspeptidase.

Coexisting disorders: Human immunodeficiency virus [HIV] and hepatitis $\mathrm{C}$ virus infection, alcoholic liver disease and hepatitis $C$

Overlap syndrome: Primary biliary cirrhosis with autoimmune hepatitis.

Fatty liver: liver biopsy can distinguish between benign steatosis and progressive steatohepatitis;

Space replacing processes discovered by chance during a routine imagistic check up.

Periodic needle biopsy is also valuable in the management of a few diseases.

In autoimmune hepatitis, monitoring the plasma cell score on liver biopsy may help predict relapse;

Liver transplantation: possible complications occurring after the liver transplant rejection or the disease recurrence (Carey \& Carey, 2010).

\subsection{The liver biopsy interpretation}

Histologic assessment of a liver biopsy specimen remains the "gold standard" for quantifying fibrosis that is essential to guide management and predict prognosis in patients with chronic liver injury .

There is a wide range of methods used to interpret a liver biopsy. For morphological analysis of liver liver biopsy is necessary to use either ordinary staining (hematoxilin-eozin), or special ones: Masson's trichrome stain (for fibrosis), Pass stain (for glycogen), Pearls stain (for intrahepatocyte iron deposits) and rhodamin (for hepatocyte copper). Immunohistochemical and in situ mRNA hybridization methods to identify specific matrix components can be employed for experimental aim and have some utility in assessing fibrosis progression.

The most important role of liver biopsy is the assessment of necroinflammation (grade) and fibrosis (stage). The morphological features of chronic viral hepatitis are portal fibrosis, portal and periportal mononuclear inflammation and necroinflammatory changes within the parenchyma with a an irregular distribution between one lobule and another. There is also regenerative activity in an irregular distribution. The degree of necrosis depends on the disease activity -more severe during exacerbations and lesser in quiescent stages. Fibrosis progresses to cirrhosis and the periseptal inflammatory activity continues with cirrhosis .

A number of numerical scoring systems have been used to evaluate the amount of inflammation and degree of fibrosis in liver biopsy specimens from patients with chronic liver disease. Histological scores such as Metavir, Knodell, Ishak and Desmet/ Scheuer systems were created to standardize the interpretation of liver biopsies. The Knodell system was the first staging system, having a total of 18 for necroinflammation and a total of 4 for fibrosis (0-4). However, this system is noncontiguous and no longer used. Instead, the Ishak system had more details for necroinflammation (0-18) and expanded scores for fibrosis(0-6). This may be helpful in clinical studies when fine gradations of fibrosis pattern are required to demonstrate change, but this system is not commonly used in clinical practice. These scores are semi- quantitative scales for evaluation of a complex pathobiological process: fibrosis, necroinflammation and finally nodule formation. The Metavir system is simpler with a combination score for inflammation including periportal and lobular activity (A0-A3) 
and a fibrosis score on a scale of F0-F4. Metavir is the most used system in clinical practice today. The accuracy of grading and staging is dependent on the adequacy of the specimen and the experience of the pathologist.

Bedossa considers that the advantages of using the scores for liver biopsy interpretation consist in :an increase of reproducibility between pathologists, a standardization of biopsies reports, an homogeneity between liver centers, and a better comparison of consecutive biopsies for monitoring disease follow-up. The scores also serve as reference for choosing and validation of non invasive methods for liver fibrosis assessment.

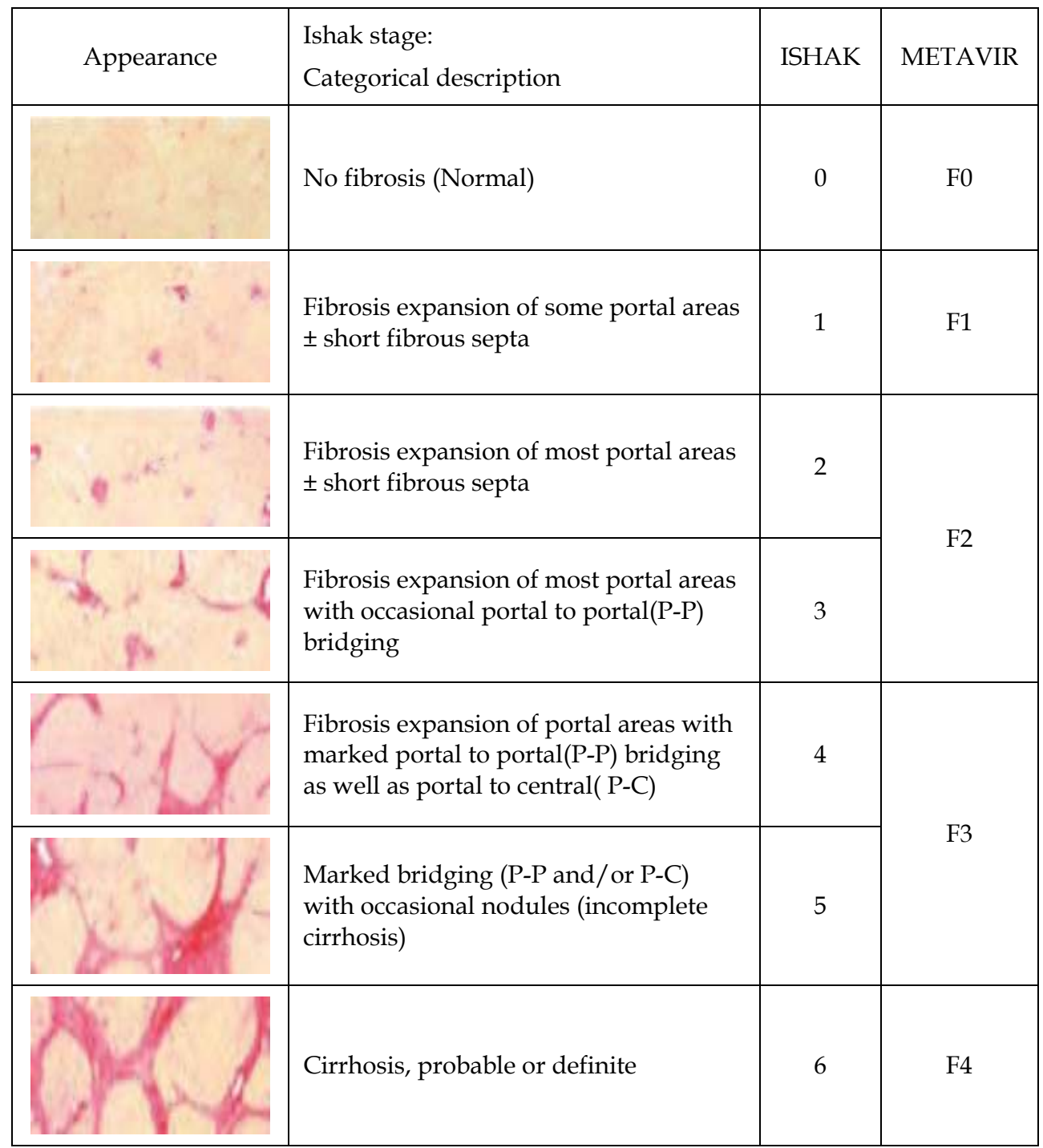

Table 1. Scoring systems in liver fibrosis.( Standish RA et al.,2006) 


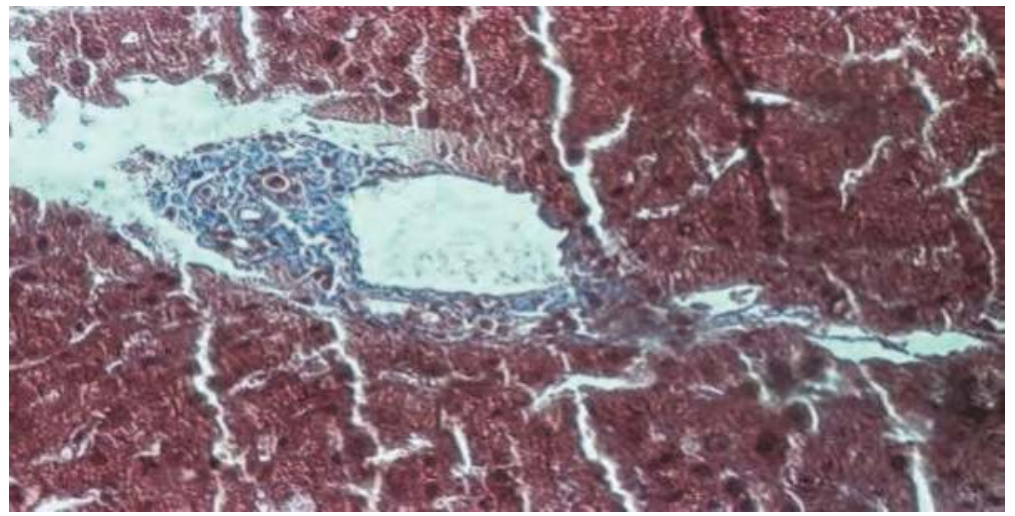

Fig. 1. Fibrosis stage 1

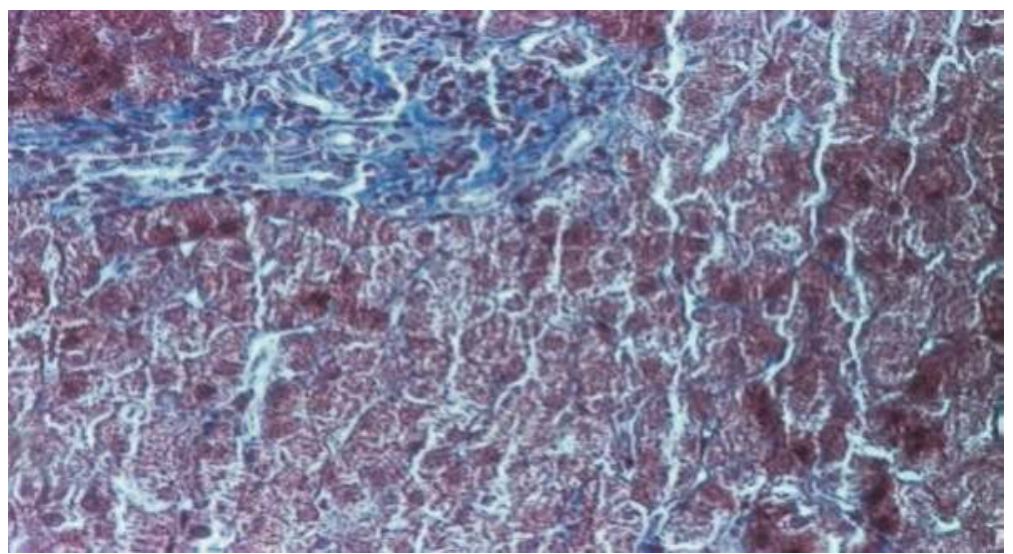

Fig. 2. Fibrosis stage 2

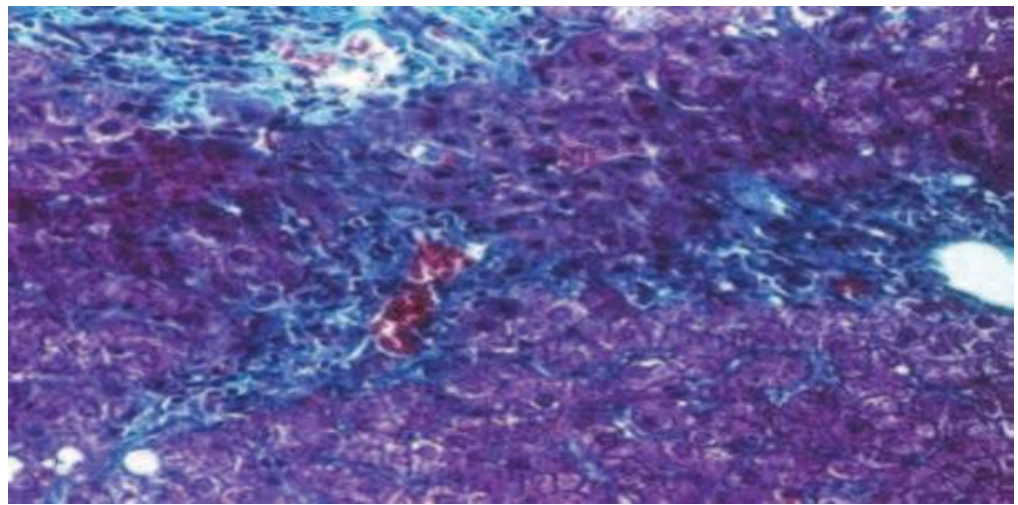

Fig. 3. Fibrosis stage 3. 


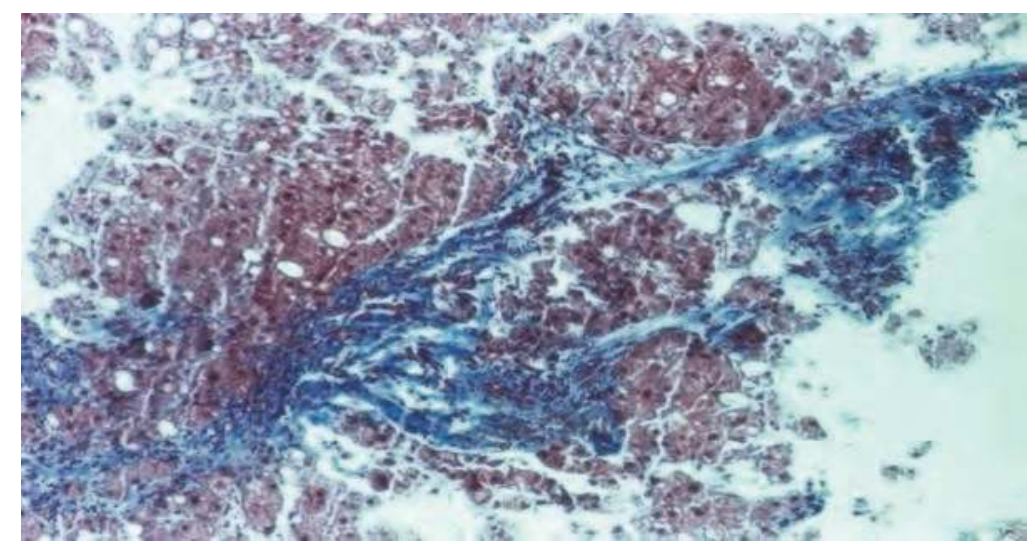

Fig. 4. Fibrosis stage 4

\subsection{Complications of liver biopsy}

Liver biopsy is a safe procedure if it is carried out by experienced gastroenterologists. Froehlich et al noticed a lower rate of complications in the case of doctors who performed over 50 biopsies in a year. Although the liver is a highly irrigated organ, complications after the biopsy are rarely. Thus, it was noted that over $60 \%$ of the complications occur within two hours and over $90 \%$ within the first 24 hours after the procedure. Within out patients who present complications, $1-3 \%$ require hospitalization, often after Tru-cut type needle use.

Complications may be minor (pain or vagal reactions, blood hypotension) or major. Significant complications, defined as those resulting in hospital admission or prolonged hospitalisation, occur in 1-5\% of patients. The mortality rate is reported between 1:1000 and 1:10000. The complications of liver biopsy and their frequency are indicated in table no. 2

\subsection{Limits of liver biopsy}

The hepatic function is compromised to a minimum until the development of cirrhosis. The presence of clinical manifestations such as ascites and encephalopathy are clear evidences of the advanced disease.

So far, only liver biopsy has been able to determine the severity of pre-cirrhotic stages. However, the role of liver biopsy as a "golden standard" in diagnosing liver diseases seems to be controversial due to the development of modern imagistic techniques: ultrasonography, computer tomography, nuclear magnetic resonance and the appearance of noninvasive serum markers.

Liver biopsy may be the "golden standard" in assessing liver fibrosis, but it is not 24 karat gold. We have to mention that the biopsy needle takes a small fragment from the liver. It is important to take into account that a biopsy is merely a sample of the entire liver and the scarring in chronic liver disease is typically irregularly distributed in the liver. For this reason, the perfect liver biopsy should remove a fragment into a known area of the disease or multiple fragmentes in many areas of a diseased liver. 


\begin{tabular}{|c|c|}
\hline \multicolumn{2}{|l|}{ Complications of liver biopsy } \\
\hline Pain at the right hypochondrium, shoulder & $0.056-83 \%$ \\
\hline \multicolumn{2}{|l|}{ Arterial hypotension } \\
\hline Hemorrhagic complications & $\begin{array}{l}\text { sub capsular hematoma: } 0.05 \% \\
\text { intrahepatic hematoma: } 0.05 \% \\
\text { intraperitoneal bleeding: } 0.03 \% \\
\text { hemobilia: } 0.05 \%\end{array}$ \\
\hline Bacteriemia & $0.08 \%$ \\
\hline Death & $0.001-0.0001 \%$ \\
\hline Bile peritonitis & $0.03-0.22 \%$ \\
\hline Pneumothorax, hemothorax & $0.08-0.28 \%$ \\
\hline Subcutaneous emphysema & $0.014 \%$ \\
\hline \multicolumn{2}{|l|}{ Subphrenic abscess } \\
\hline \multicolumn{2}{|l|}{ Anaphylactic shock (hydatid cyst) } \\
\hline Break of the biopsy needle & $0.02 \%$ \\
\hline Biopsy of other organs & $\begin{array}{l}\text { lungs- } 0.001 \% \\
\text { bile }-0.003 \% \\
\text { colon- } 0.003 \% \\
\text { kidneys- } 0.09 \%\end{array}$ \\
\hline
\end{tabular}

Table 2. The complications of liver biopsy

The liver biopsy removes only about $1 / 50000^{\text {th }}$ of the liver and can miss the diagnosis of cirrhosis in $20 \%$ to $30 \%$ of patients. That is why, many investigators recommend a biopsy length at least $15 \mathrm{~mm}$, containing sixt or eight portal tracts, to improve the diagnostic accuracy. Both the size of the biopsy and the number of biopsies taken have a major effect on diagnosis accuracy. Although widely used, liver biopsy can be a source of errors given by the small size of the samples or the inappropriate sizes of the needle.The length of the fragment is proportional to the length of the needle used (18 gauge) and the size of the liver fragment is essential in assessing the hepatic architecture. There are several studies which revealed a decrease of diagnosis accuracy in biopsies smaller than $2 \mathrm{~cm}$. In a study which comprised 10.000 virtual biopsies, Bedossa and collaborators showed that the staging of liver fibrosis is accurate in $65 \%$ of the cases when the analyzed biopsy fragment was at least $15 \mathrm{~mm}$ long, in $75 \%$ of the cases when the fragment was at least $25 \mathrm{~mm}$ length and that the best length should be $40 \mathrm{~mm}$. The same study, in which laparoscopic biopsies were collected from both lobes of patients with chronic hepatitis $C$, estimated that biopsy specimens with a minimum $4 \mathrm{~cm}$ or greather in length would reliably avoid sampling error. However, in many specialized hepatology centers, the biopsy fragment does not meet the best criteria for an accurate histological analyse. Surgical wedge biopsy provides adequate tissue volume to overcome this problem. 
Another limitation of the liver biopsy is that is very sensitive to analysis errors. Several studies have investigated the interobserver and intraobserver variability in the histological diagnosis of liver diseases. To minimize the analysis errors and to standardize the histological evaluation, staging scores for fibrosis, such as the METAVIR, Ishak and Scheuer systems, were created. Nevertheless, liver biopsy is associated with error in fibrosis staging in up to $20 \%$ of patients and a misdiagnosis of cirrhosis in up to $15 \%$ of patients. In example of the resulting sampling errors that can occur, were considered the two most common chronic liver diseases: hepatitis $C$ and fatty liver disease. Regev et al (2002) performed laparoscopically guided biopsy of the right and left hepatic lobes in a serie of 124 patients with chronic hepatitis C. The two fragments of the same patient were interpreted by the same anatomopathologist who was not awared about the identity of the biopsy fragment. Differences of more than one Metavir stage of fibrosis was present in $33,1 \%$ of cases and more than 2 stages in $2.4 \%$. In $14.5 \%$, cirrhosis was diagnosed in one lobe but not the other. As regards liver diseases of other etiologies than the viral one, differences were even bigger. In a study on patients with nonalcoholic fatty liver disease, Ratziu et al found that differences of more than one stage Metavir was present in approximately $40 \%$ of paired liver biopsy; in the patients with primary sclerosing cholangitis or primary biliary cirrhosis, $\geq 1$ fibrosis stage discordances occurred in up to $60 \%$ of paired liver biopsy.

Clearly, needle liver biopsy is far from an ideal test. For these reasons it is necessary to develop some semi-automated systems to assess additional histological and immunohistochemical features.

Another limit of the liver biosy is related to slow rate of fibrosis progression; from normal liver to cirrhosis it takes more than 20 years. Follow-up biopsy is too insensitive to detect changes in fibrosis progression or regression within weeks to months and even years.

From another point of view, liver biopsy is an invasive procedure with certain unavoidable risks and complications. For both, the physician and the patient, the decision to proceed with liver biopsy is not a trivial one. There is an urgent need for a noninvasive diagnostic procedure for liver fibrosis because this is currently the main limitation of antifibrotic drug testing in clinical trials.

\section{Noninvasive methods for the evaluation of liver fibrosis}

Liver biopsy has been used in more than a century for the staging and diagnosis of acute or chronic liver diseases. In order to monitor patients with chronic liver diseases, liver biopsy has been increasingly less used because of the sampling and interpretation errors, the patient's high emotional cost and especially because it captures only one moment in a diffuse and evolutive hepatic process. In this context, the identification of a noninvasive method for the evaluation of liver fibrosis is a challenging option.

Noninvasive testes are an attractive alternative to liver biopsy for staging and monitoring of chronic liver diseases. A noninvasive method which provides the same information as liver biopsy is also desirable in cases when the latter is not possible.

The purpose of any noninvasive technique is to replace liver biopsy. These methods have the advantage of representing the liver in its entirely unlike puncture which analyzes only a limited fragment of liver. They also provide information related to the hepatic function: secretion, bile excretion or endothelial uptake. 
Considering the slowly evolution of chronic liver diseases, the information provided through liver puncture is not enough to monitor the disease at short time intervals ue to the minimal histological changes. Noninvasive tests have to be sensitive enough to detect the minor changes of the dynamic fibrosis process.

Another indication of noninvasive tests is that they evaluate the efficiency of antifibrotic treatments, which cannot be performed through repeated hepatic punctures. It is important to have safe and efficient methods to objectify liver fibrosis from several perspectives. In the first place, patients with liver fibrosis are part of a high risk group as regards liver diseases mortality. In the second place, the prognosis of chronic liver diseases depends on the severity and related risk factors. In the third place, when the etiologic factor can be removed, it is important to introduce the antifibrotic therapy as soon as possible to reverse the hepatic lesions.

The criteria used in evaluating the severity of liver diseases through other procedures than liver biopsy are not specified yet. That is why, for now, potential noninvasive methods must be relatively well correlated with the biopsy. The errors of this histological method prevent the perfect correlation between the biopsy and noninvasive tests in the diagnosis and staging of liver fibrosis. The best correlation occurs in biopsies $>$ de $2.5 \mathrm{~cm}$, the correlation index $(\mathrm{r})$ being 0.85 . For the interpretation of noninvasive tests, it is important to consider the fact that liver biopsy may fail in $20 \%$ of the cases, especially for intermediary forms of liver fibrosis. This is the reason why many experts believe that the noninvasive methods whose ROC (Receiver Operator Characteristic) curve is $0.85-0.90$ are as effective as the histological exam in the accurate staging of liver fibrosis.

The two major categories of noninvasive tests include: serum fibrosis markers and hepatic elasticity imaging (elastography).

The results of noninvasive tests will be interpreted in the clinical context; in case of discrepancies, liver biopsy is recommended.

\subsection{Serum fibrosis markers}

The physiopathology's knowledge of liver fibrosis has determined a high number of biomarkers used to diagnose it. Depending on the elements they represent, there are two marker categories: direct (representing components of extracellular matrix) or indirect (reflecting hepatic inflammation and function).

In order to be sensitive and specific, therefore ideal for the diagnosis of liver fibrosis, Afdhal N.H (2007) considers that biomarkers have to meet the following requirements:

- they should be specific hepatic tests;

- the results should not be influenced by the alteration of the hepatic, renal or reticuloendothelial function;

- they should reflect fibrosis regardless of etiology;

- $\quad$ they should be reproducible and easy to perform;

- they should be sensitive enough to differentiate among various fibrosis stages;

- the risks for patients should be minimum;

- the cost should be low.

Due to the high number of molecules involved in liver fibrosis efforts were made to compile the serum markers within panels for clinical use. More indicators and sophisticated scores utilizing direct and indirect markers have been developed to enhance the detecting and noninvasive staging of liver fibrosis. The most widely known of these are listed in table no.3. 


\begin{tabular}{|c|c|c|c|c|c|}
\hline Panel & Liver Disease & Se & Sp & PPV & NPV \\
\hline $\begin{array}{c}\text { AST/ALT } \\
\text { ratio }\end{array}$ & AST/ALT & $53 \%$ & $100 \%$ & $100 \%$ & $81 \%$ \\
\hline Forns test & Platelets, GGT, Cholesterol & $94 \%$ & $51 \%$ & $40 \%$ & $96 \%$ \\
\hline APRI Index & AST, Platelets & $41 \%$ & $95 \%$ & $88 \%$ & $64 \%$ \\
\hline PGA Index & Platelets, GGT, apo A & $91 \%$ & $81 \%$ & $85 \%$ & $89 \%$ \\
\hline FibroTest & $\begin{array}{c}\text { GGT, haptoglobin, bilirubin, apoA, } \\
\text { alpha2-macroglobulin }\end{array}$ & $87 \%$ & $59 \%$ & $63 \%$ & $85 \%$ \\
\hline Fibrospect & $\begin{array}{c}\text { Hyaluronic acid, TIMP-1, alpha } 2- \\
\text { macroglobulin }\end{array}$ & $83 \%$ & $66 \%$ & $72 \%$ & $78 \%$ \\
\hline FPI & AST, Cholesterol, HOMA-IR & $85 \%$ & $48 \%$ & $70 \%$ & $69 \%$ \\
\hline ELF & ECM proteins and proteinases & $90 \%$ & $41 \%$ & $35 \%$ & $92 \%$ \\
\hline
\end{tabular}

Abbreviations: Se, sensitivity, Sp, specificity, PPV, positive predictive value, NPV, negative predictive value, AST, aspartate aminotransferase, ALT, alanine aminotransferase, GGT, Gamma glutamyl transferase, apoA, apolipoprotein $\mathrm{A}_{1}$, TIMP-1, tissue inhibitors of metalloproteinase 1, HOMA-IR, homeostasis model assessment of insulin resistance, EMC, extracellular matrix molecules

Table 3. Diagnostic performances of serum markers panels for hepatic fibrosis compared to the referenced standard of liver biopsy (Adapted from Rockey DC\& Bissel DM, 2006).

The serum markers of fibrosis are an attractive, noninvasive and affordable alternative, replacing at least part of the liver biopsies used in clinical practice. Crockett et al (2006) made a comparison between liver biopsy and serum markers, considering the advantages and disadvantages of the two methods used alternatively to diagnose and stage liver fibrosis.

\begin{tabular}{|l|l|l|}
\hline Factor & Liver biopsy & Serum markers \\
\hline Cost & 2200\$ & Laboratory cost \\
\hline Risks & Significant & Minimal \\
\hline Contraindications & $\begin{array}{l}\text { Multiple: bleeding diathesis, morbid } \\
\text { obesity, ascites, extrahepatic biliary } \\
\text { obstruction }\end{array}$ & $\begin{array}{l}\text { Conditions with high rate of } \\
\text { false positivity }\end{array}$ \\
\hline $\begin{array}{l}\text { System } \\
\text { requirements }\end{array}$ & $\begin{array}{l}\text { Operator, pathology laboratory, } \\
\text { pathologist }\end{array}$ & $\begin{array}{l}\text { Clinical laboratory, phlebo- } \\
\text { tomy, materials. }\end{array}$ \\
\hline $\begin{array}{l}\text { Specimen } \\
\text { adequacy }\end{array}$ & $\begin{array}{l}\text { Length of liver fragment at least 15 } \\
\text { mm with 6-8 portal tracts }\end{array}$ & Blood sample \\
\hline False positives & Interobserver variability & $\begin{array}{l}\text { Sepsis, nonhepatic infla- } \\
\text { mmation, hemolysis, trom- } \\
\text { bocitopenia }\end{array}$ \\
\hline False negatives & Sampling variability & Varies per test \\
\hline Time for results & 24-72 hours minimum & $1-2$ hours minimum \\
\hline
\end{tabular}

Table 4. Comparison of liver biopsy and serum markers of fibrosis

The ability of serum tests of fibrosis are similar, with area under the receiver operating characteristic curve 0.8-0.85. Compared to biopsy, current serum biomarkers represent the whole liver, permit only crude staging sparing about $30-40 \%$ patients for biopsy and reflect 
also liver function (secretion, endotelial uptake).Although their accuracy value are improving they cannot supplant direct analysis of the liver. They have some limitatios:

- They reflect the rate of matrix turnover and tend to be more elevated when there is high inflammatory activity;

- None of the molecules is liver specific, so concurrent sites of inflammation may contribute to serum level;

- $\quad$ Serum levels are influenced by clearance rates;

The clinical usefulness of serological tests is to set quickly whether fibrosis is mild or severe, being less efficient in intermediary fibrosis stages. Most serum markers are more efficient in identifying fibrosis that in infirming it. That is why, when fibrosis is severe, regardless of the invasive or noninvasive diagnosis method, patients without signs of portal hypertension should start etiologic treatment.

So far, there is no ideal noninvasive test. Serum markers are based on probability calculations and thus they are less specific. Their diagnosis performances increase through the combined use of several serum markers tests.

Using the algorithms based on the sequential combination of serum markers, diagnosis accuracy in detecting significant fibrosis can increase to $93-95 \%$, and the liver biopsies could be avoided in $50 \%$ of the patients with chronic liver diseases. As clinical experience accumulates we shall expect that serum markers will begin to replace many of the liver biopsies used today for prognostic and treatment purpose.

Are serum markers ready for the first line fibrosis diagnosis? Overall, the serum assay approach remains promising, in part because these tests may represent an "integrated readout of liver activity rather than a minute sampling of the type obtained by conventional liver biopsy. Initially established as a niche especially in patients who have a high risk of complications from liver biopsy, by continued refinement, biomarkers are very close to become first line method for the diagnosis and monitoring of liver fibrosis. (Schiff, 2007).

\subsection{Elastography imaging}

Elastography is a much simpler method used to stage liver fibrosis than the $\mathrm{X}$ ray methods which are commonly used. This technique is quick and noninvasive. It helps to determine the stiffness of the hepatic tissue. It is well-known that the stiffness of the hepatic tissue is closely related to the stage of liver fibrosis. Touching the firm edge of the liver is a clinical parameter used for many years to diagnose cirrhosis.

\subsubsection{Transient Elastography (TE)}

Fibro scan is a device which measures hepatic stiffness through transient elastography. It determines the elasticity of a hepatic volume equal to a cylinder with the diameter of $1 \mathrm{~cm}$ and the length of $4 \mathrm{~cm}$, being thus 100 times larger than the standard biopsy fragment and, as such, more representative for the entire hepatic parenchyma.

Fibro scan uses an ultrasound transducer fixed on the axes of a vibrator which induces a low frequency vibration $(50 \mathrm{MHz})$. The vibration transmitted to the hepatic tissue produces an elastic wave which propagates inside the liver. The propagation of this wave is followed by an ultrasound impulse and the measurement of its speed determines, through direct correlation, the tissue stiffness. The more rigid the liver, the higher the propagation speed. The results are expressed as kilopascal. Since a larger area of hepatic tissue is studied, 
diagnosis errors related to the sample size which occur in standard liver biopsy are eliminated, even in disorders with focal liver fibrosis.

This technology has many of the features of a noninvasive ideal method in evaluating liver fibrosis, with a series of advantages: it is quick, reproducible, painless and safe.

Transient elastography is a relative new method. The first clinical data related to this technology were published in 2002. Numerous studies on patients with chronic hepatitis C demonstrated that elastography is a safe method for detecting severe fibrosis (Casterra, 2010). Although it is limited in differentiate the intermediary fibrosis stages, its major advantage is that it accurately detects persons with significant fibrosis.

Usually, 10 measurements are carried out for each patient, the total exam duration being 510 minutes. The success rate is automatically calculated by the machine as a ratio between the number of successful measurements and the total number of measurements. The recommendations of the manufacturers of this technology are that transient elastography can only be validated if there are 10 measurements with a success rate of at least $60 \%$. However, recent studies have suggested that 3 validated measurements have the same value as 10 measurements for cirrhosis diagnosis; the minimum number of measurements for significant fibrosis has still to be set. It is considered that 10 validated measurements and a success rate of at least $60 \%$ can be obtained in $90 \%$ of exams.

Transient elastography is easy to interpret; the coefficient of concordance for the same examiner is $96-98 \%$ (the correlation coefficient in the same class) and between examiners, the coefficient of concordance is of $89-98 \%$. Measurements validation depends on the IQR (interquartile range), $<30 \%$ ) and the success rate of measurements, $>60 \%$.

The largest study of hepatic elastography evaluated 327 patients with chronic infection C (Ziol et al, 2005) . For each patient, fibrosis was staged by means of 2 methods: Fibro scan and liver biopsy. Metavir scoring system for histological staging of liver fibrosis served as reference. The conclusion was that elastography is a reliable tool to detect significant fibrosis or cirrhosis. Table no. 5 indicates the diagnosis performance of Fibro scan in detecting significant fibrosis, as well as the authors who reported these results.

\begin{tabular}{|c|c|c|c|c|c|c|}
\hline Study & $\begin{array}{c}\text { Liver } \\
\text { disease }\end{array}$ & $\begin{array}{c}\text { No } \\
\text { patients }\end{array}$ & $\begin{array}{c}\text { Prevalence of } \\
\text { significant } \\
\text { fibrosis }\end{array}$ & AUROC & $\begin{array}{c}\text { Limit } \\
\mathbf{K P a}\end{array}$ & Se/Sp \\
\hline $\begin{array}{c}\text { Gomez- } \\
\text { Dominguez et al. }\end{array}$ & Mixt & 94 & 82 & 0.74 & 4.0 & $94 / 33$ \\
\hline Fraqelli et al & Mixt & 200 & 50 & 0.86 & 7.6 & $81 / 76$ \\
\hline Chang et al & Mixt & 120 & 44 & 0.86 & 9.0 & $83 / 85$ \\
\hline Castera et al & HCV & 183 & 74 & 0.83 & 7.1 & $67 / 89$ \\
\hline Ziol et al & HCV & 251 & 65 & 0.79 & 8.8 & $56 / 91$ \\
\hline Yoneda et al & NAFLD & 67 & 49 & 0.87 & 6.6 & $83 / 81$ \\
\hline
\end{tabular}

Abbreviations: HCV, hepatitis virus C, Se, sensitivity, Sp,specificity, NAFLD - non alcoholic fatty liver disease

Table. 5. Fibroscan performance in detecting significant fibrosis (Guha \& Rosenberg,2008)

When comparing with liver biopsy, TE is non-invasive, easy to repeat, reproducible, without contraindications, highly performant technique for detecting cirrhosis. Its disadvantage is that unlike liver biopsy, TE is unable to discriminate between intermediate 
stages of fibrosis. Comparing with serum markers, TE has lower applicability: failure in $5 \%$ of cases and unreliable results in 15\% of cases.( Casterra, 2010). The known limits of this method are: the impossibility to conduct measurements in case of ascites (elastic waves do not propagate in liquids) and in patients with narrow intercostals spaces (it is necessary to insert the probe in the intercostals space). Another significant limiting factor is obesity (body mass index $>28 \mathrm{Kg} / \mathrm{m}^{2}$ ) due to the diminishing of the surface fat tissue signal. False positive results appear in case of acute hepatitis, extra hepatic cholestasis and congestive heart failure (Casterra, 2010). The combination between TE and serum markers increases diagnostic accuracy for liver fibrosis.

\subsubsection{Magnetic Resonance Elastography}

Magnetic resonance elastography (MRE) has the same basic principles as sonographic elastography. By using a probe (pneumatic or electromagnetic), placed directly on the patient's abdominal wall, a shear wave is created. A specialized system of magnetic resonance measures the sequential propagation of the shear wave and transforms the data analyzed as an elastogram (color - encoded images). The analysis contains the liver in its entirety and allows assessing of fibrosis distribution, which is usually heterogeneous. Although until now the number of publications related to this method has been reduced, the results look promising. A study which compared MRE with the APRI index in patients with viral or alcoholic liver diseases has shown that MRE is more sensitive in detecting moderate and severe fibrosis than the APRI index. Among 96 patients with liver biopsy and noninvasive examinations the reproducibility of both TE and MRE were excellent. The elasticity imaging techniques had greater diagnostic accuracy than APRI for detecting liver fibrosis. MRE was also found to be more accurate than TE for significant fibrosis $(F \geq 2)$ and for cirrhosis diagnosis (Huwart 2008).

The problems related to this technique refer to the increased exposure time (the exam takes 60 minutes), the significant capital invested in the equipment and the computerized analysis system, the standardized measurement threshold and method reproducibility. In contrast with TE, MRE can be successfully performed in obese patients (BMI $>40 \mathrm{~kg} / \mathrm{m}^{2}$ ). Furthermore, MRE had in part higher diagnostic accuracy in detecting significant fibrosis $(\mathrm{F} \geq 2)$ as compared with TE by examining of larger liver parenchyma areas (Huwart, 2008).

Although modern magnetic resonance technique is not the most pragmatic noninvasive methods it is a promising method as regards the diagnosis potential of liver fibrosis. The acknowledgment of its diagnosis sensitivity still requires large population studies.

\subsubsection{Acoustic Radiation Force Imaging (ARFI)}

A new technique used to determine liver elasticity is ARFI elastography. The elastographic analysis model combines two technologies, 'Virtual Touch tissue imaging ${ }^{\mathrm{TM}}$ ' and 'Virtual Touch tissue quantification ${ }^{\mathrm{TM}}$ ', which allow the quantitative and qualitative analysis of liver elasticity. Using the Virtual Touch application, the tissue gets compressed via acoustic energy. It enables the physician to evaluate the mechanical characteristics of deep tissue and tissue changes. ARFI imaging (Virtual Touch tissue quantification ) measures liver stiffness within a defined region of interest (central window of $5 \mathrm{~mm}$ axial by $4 \mathrm{~mm}$ width), while performing real-time B-mode imaging. Transmission of short-duration ( 262 sec) longitudinal acoustic pulses with a fixed transmit frequency of $2.67 \mathrm{MHz}$ leads micron-scale displacements in liver tissue, which results in a shear -wave propagation, away from the 
region of excitation. Maximum movement is estimated by ultrasound detection fascicles adjacent to the central acoustic pulse. By measuring the maximum movement amplitude as compared to the time of each side fascicle, the shear - wave speed of the tissue can be reconstructed. Results expressed in meters per second are displayed by the machine (Friedrich-Rust, 2009).

Preliminary results indicate that ARFI imaging technology is a promising method for the diagnosis of significant fibrosis in patients with chronic hepatitis C.( Friedrich-Rust, 2009, Fierbinteanu-Braticevici, 2009). The benefits of ARFI imaging as compared with Fibro scan consist in: lesser time consuming (new software allowed to the 12 measurements per $1 \mathrm{~min}$ ) and successful measurements in difficult patients (obese) because of subcostal scanning. Another advantage of ARFI imaging is that it is integrated into a conventional ultrasonography (US) system and can thus be performed during standard US examinations of the liver, which are routinely performed in patients with chronic liver disease. Further studies on large number of patients are needed in order to specify the role of ARFI elastography for noninvasive staging of liver fibrosis.

\subsection{Breath tests}

Taking into account that the liver has a complex and high metabolic capacity, translated into detoxification, synthesis, metabolization, storage, a unique classical test may not reflects all these sides. More, each function may be different affected in various stages of the liver disease, so that the determination of the reserve capacity of the liver appears to reflect a global functional image. During the last decade, several dynamic tests have been proposed to improve the functional diagnosis of liver diseases. Although numerous tests which explore a specific hepatic function have been used, these methods were not introduced in the clinical practice.

The introduction of breath tests that use marked carbon $\left(\mathrm{C}^{13}\right)$ attached to various substrata (aminopyrin, phenylalanin, methacetine, phenacetyne, galactose) was due to the need to have a quick and practical test to determine the functional hepatic reserve. While neither of these tests allows quantification of a global liver function, each has been shown to reflect the most important partial liver functions i.e. cytosolic, microsomal or mitochondrial function.

The principle of breath tests is simple. An ingested substrate attached to $\mathrm{C}^{13}$ or $\mathrm{C}^{14}$, administered orally or parenterally is converted by the liver in ${ }^{13} \mathrm{CO}_{2}$ or ${ }^{14} \mathrm{CO}_{2}$. The marked $\mathrm{CO}_{2}$ is collected at different time intervals in an alkaline environment which serves as a trap. The hepatic metabolism of the bonding substrate may be determined from a semi quantitative perspective by multiplying the specific $\mathrm{CO}_{2}$ activity in the exhaled air within a given time interval. An ideal compound for this purpose is metabolized solely by the liver and therefore reflects liver function.

In the beginning, breath tests required sophisticated and expensive mass spectrometers that were no longer used. ${ }^{13} \mathrm{C}$ can now be measured with non dispersive infrared spectrometry, a simple and cost-saving method which provides information correlated with those of the mass spectrometry. Functional hepatic tests, including breath tests, together with clinic scores, had been proved to be helpful in diagnosis and prognosis of the patients with advanced hepatic disease. As regard the role of methacetin breath test (MBT) in the assessement of fibrosis in patients with chronic hepatitic $C$, our study indicated that the methacetin breath test parameters didn't allow differentiation of patients without fibrosis from those with mild fibrosis (F1). This limit was overcome by the fact that MBT was highly 
correlated with significant fibrosis $(\geq$ F2) which is considered the hallmark of progressive liver disease and a stronger indication for treatment.

Additional studies are required to assess the role of these tests as an alternative to liver biopsy in the diagnosis and follow-up of hepatic injury in patients with chronic liver diseases.

\section{Conclusion}

Liver biopsy remains an important tool in the evaluation and management of liver disease. However it is invasive, can cause significant complications and clearly, needle liver biopsy is far from an ideal test. Even though it is an imperfect "gold standard", liver biopsy remains an important tool in the evaluation and management of liver disease.

For this reason, the efforts to estimate the hepatic lesion stage through noninvasive methods are justified. Noninvasive investigations, such as various biomarkers, fibrosis scoring panels, imaging techniques and breath tests offer considerable promise in their ability to detect and to stage liver fibrosis. Until now, not even one noninvasive test can be considered enough sensitive to predict by itself the severity of acute or chronic liver diseases. That is why combining various noninvasive tests is promising and many times they can avoid liver biopsy. Further testing and validation are needed for these noninvasive procedures to refine their role of clinical practice and supplant the need for liver biopsy in chronic liver diseases. Now, when many non - invasive procedures have been studied, the question is if that do we still need a liver biopsy? We think that the answer is "yes", because despite its limitations, the liver biopsy remains an invaluable procedure of the clinician, whenever the etiology of liver disease is in impass. Liver biopsy is still needed if laboratory testing and imaging studies are inconclusive. It may take many years, if at all, until liver biopsy will be fully replaced.

\section{References}

Carey E , Carey W. (2010). Noninvasive tests for liver disease, fibrosis, and cirrhosis: Is liver biopsy obsolete? Cleaveland clinic journal of medicine, 77 (8), 527. ISSN: 0891-1150

Castera I, Pinzani M. (2010). Biopsy and non-invasiv e methods for the diagnosis of liver fibrosis does it take two to tango?, Gut, 59, 861-6, ISSN: 0017-5749

Friedrich-Rust M, et al. (2009). Liver Fibrosis in Viral Hepatitis: Noninvasive Assessment with Acoustic Radiation Force Impulse Imaging versus Transient Elastography. Radiology, 597-604, ISSN 0033-8419

Fierbinteanu-Braticevici C, Andronescu D, Usvat R,et al .(2009). Acoustic radiation force imaging sonoelastography for noninvasive staging of liver fibrosis. World Journal of Gastroenterology, 15, 5525-5532, ISSN: 1007-9327

Huwart L, et al. (2008). Magnetic resonance elastography for the noninvasive staging of liver fibrosis. Gastroenterology,135, 32-40, ISSN: 0016-5085

Knodell RG, Ishak KG, et al. (1981). Formulation and application of a numerical scoring system for assessing histological activity in asymptomatic chronic active hepatitis. Hepatology,1, 431-435, ISSN, 1665-2681

Metavir Cooperative Study Group. (1994). Intraobserver and interobserver variations in liver biopsy interpretation in patients with chronic hepatitis $C$. The French METAVIR Cooperative Study Group. Hepatology,20, 15-20, ISSN: 1665-2681 
Schiff E, SorrellM, Maddrey W, (2007). Schiff's diseases of the liver,LippincottWilliams\&Wilkins, 10th edition ISBN, 10-7817-60-40-2,Philadelphia,USA

Desmet VJ, Scheuer PJ et al. (1994) Classification of chronic hepatitis: diagnosis, grading and staging. Hepatology, 19, 6, 1513-20, ISSN: 1665-2681

Standish R. A. et al, (2006) An appraisal of the histopathological assessment of liver fibrosis Gut, 55, 569-578, ISSN: 0017-5749

Bedossa P.(2011). How pathological scores can be improved?. EASL, Monothematic Conference, Nice.

Regev A et al. (2002). Sampling error and intraobserver variation in liver biopsy in patients with chronic HCV infection. American Journal of Gastroenterology, 97, 2614-2618, ISSN: 0002-9270

Ratziu V et al. (2005). Sampling variability of liver biopsy in nonalcoholic fatty liver disease.Gastroenterology, 128, 7, 1898-906 ISSN: 0016-5085

Afdhal N.H.(2007).Non-biopsy methods to determine hepatic fibrosis, Postgraduate Course, AASLD, 91-94

Rockey DC, Bissell DM. (2006) Noninvasive measures of liver fibrosis. Hepatology. 43,2, Suppl 1, S113-20. ISSN: 1665-2681

Ziol M, et al. (2005). Noninvasive assessment of liver fibrosis by measurement of stiffness in patients with chronic hepatitis C. Hepatology, 41,1, 48-54,ISSN: 1665-2681

Guha IN. Rosenberg WM. (2008).Noninvasive Assessment of Liver Fibrosis: Serum Markers, Imaging, and Other Modalities. Clinical Liver Disease, 12, 883-900, ISSN : 1590-8658

Crockett S.D. et al (2006). Do will still need a liver biopsy? Are the serum fibrosis tests ready for prime time? Clinics in liver disease,10, 513-534, ISSN:1089-3261,ISBN:1-4160-3553-2 


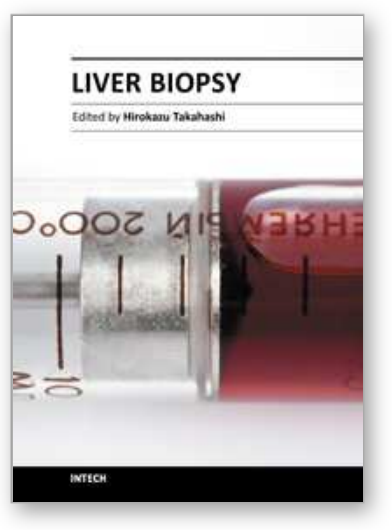

\author{
Liver Biopsy \\ Edited by Dr Hirokazu Takahashi
}

ISBN 978-953-307-644-7

Hard cover, 404 pages

Publisher InTech

Published online 06, September, 2011

Published in print edition September, 2011

Liver biopsy is recommended as the gold standard method to determine diagnosis, fibrosis staging, prognosis and therapeutic indications in patients with chronic liver disease. However, liver biopsy is an invasive procedure with a risk of complications which can be serious. This book provides the management of the complications in liver biopsy. Additionally, this book provides also the references for the new technology of liver biopsy including the non-invasive elastography, imaging methods and blood panels which could be the alternatives to liver biopsy. The non-invasive methods, especially the elastography, which is the new procedure in hot topics, which were frequently reported in these years. In this book, the professionals of elastography show the mechanism, availability and how to use this technology in a clinical field of elastography. The comprehension of elastography could be a great help for better dealing and for understanding of liver biopsy.

\title{
How to reference
}

In order to correctly reference this scholarly work, feel free to copy and paste the following:

Carmen Fierbinteanu Braticevici, Raluca Papacocea, Laura Tribus and Anca Badarau (2011). Can We Replace Liver Biopsy with Non-Invasive Procedures?, Liver Biopsy, Dr Hirokazu Takahashi (Ed.), ISBN: 978953-307-644-7, InTech, Available from: http://www.intechopen.com/books/liver-biopsy/can-we-replace-liverbiopsy-with-non-invasive-procedures-

\section{INTECH}

open science | open minds

\section{InTech Europe}

University Campus STeP Ri

Slavka Krautzeka 83/A

51000 Rijeka, Croatia

Phone: +385 (51) 770447

Fax: +385 (51) 686166

www.intechopen.com

\section{InTech China}

Unit 405, Office Block, Hotel Equatorial Shanghai

No.65, Yan An Road (West), Shanghai, 200040, China 中国上海市延安西路65号上海国际贵都大饭店办公楼 405 单元

Phone: +86-21-62489820

Fax: $+86-21-62489821$ 
(C) 2011 The Author(s). Licensee IntechOpen. This chapter is distributed under the terms of the Creative Commons Attribution-NonCommercialShareAlike-3.0 License, which permits use, distribution and reproduction for non-commercial purposes, provided the original is properly cited and derivative works building on this content are distributed under the same license. 